\title{
Radiation Analysis of Antenna Array with Synthetic Functions
}

\author{
Wei-kang YU ${ }^{1}, \mathrm{Hu} \mathrm{YANG}^{2}$, Yan-lin $\mathrm{XU}^{3}$, Xiang $\mathrm{LIU}^{4,{ }^{*}}$ and Da PENG ${ }^{5}$ \\ 1, 2,3 National University of Defense Technology, Changsha, Hunan province, China \\ ${ }^{4}$ Hunan Institute of Science and Technology, Yueyang, Hunan province, China \\ ${ }^{5}$ Telecommunication Technology Research Institute, Chengdu, Sichuan province, \\ China
}

Keywords: Method of moment, Synthetic function, Antenna array

\begin{abstract}
Synthetic basis functions method(SBFM) is introduced to analyze the electromagnetic properties of large periodic arrays. Compared with the traditional method of moment (MoM), SBFM uses fewer high order basis functions which significantly reduces the number of unknowns, contributing to higher efficiency and lower memory cost. Thus, to deal with the radiation problem of large antenna arrays, it will be of great help to improve the performance.
\end{abstract}

\section{Introduction}

Method of Moment (MoM) is an effective and accurate computational methods to obtain the radiation and scattering characteristics in modern computational electromagnetism[1]. Conventional MoM requires a typical spatial sampling rate of $\lambda / 10$ for discretization with the implementation of low order basis functions like Rao-Wilton-Glisson (RWG) basis function. Therefore, it is hardly applied to the analysis of large scale problems because of the rapid growth of the computational complexity $\mathrm{O}\left(N^{3}\right)$ and memory cost $\mathrm{O}\left(N^{2}\right)$. To improve the MoM's computational scale and efficiency, two typical branches are proposed: 1) Accelerate the matrix-vector production in the iteration process such as fast multiple method (FMM) and its improvement multilevel fast multi-pole algorithm (MLFMA). 2) Reduce the number of unknowns by constructing the high order basis function. Characteristic basis function method (CBFM) and synthetic basis function method (SBFM) are two representatives.

In this paper, SBFM is represented to analysis the radiation properties of electric conducting antenna excited by delta gap voltage sources. The result is compared with conventional MoM and CST, which validates the great strength in memory cost.

\section{Basic Theory of SBFM}

SBFM is linear combinations of the low-order basis functions, which is defined on each sub-block of the whole structure. The current distribution is obtained by the electrical field integral equation (EFIE):

$$
\boldsymbol{n} \times i k \eta \int_{S}\left[\boldsymbol{J}\left(\boldsymbol{r}^{\prime}\right) g\left(\boldsymbol{r}, \boldsymbol{r}^{\prime}\right)+\frac{1}{k^{2}} \nabla^{\prime} \cdot \boldsymbol{J}\left(\boldsymbol{r}^{\prime}\right) \nabla g\left(\boldsymbol{r}, \boldsymbol{r}^{\prime}\right)\right] d S^{\prime}=\boldsymbol{n} \times \boldsymbol{E}^{i n c}, \quad \boldsymbol{r} \in S
$$

Eq. 1 is compactly written as:

$$
\boldsymbol{n} \times \boldsymbol{L}(\boldsymbol{J})=-\boldsymbol{n} \times \boldsymbol{E}^{i n c}(\boldsymbol{r}), \quad \boldsymbol{r} \in S
$$


Where $L$ represents a linear operator and $E$ is the imposed electric field. Discretize $J$ with a series of low basis functions

$$
\mathbf{J}=\sum_{n=1}^{N} I_{n} \mathbf{f}_{n}(\mathbf{r})
$$

In SBFM, high order basis functions called SBFs are employed in form of the linear combination of RWG functions [2].

$$
\mathbf{J}=\sum_{b=1}^{N_{S B}} x_{b} \mathbf{F}_{b}(\mathbf{r})=\sum_{b=1}^{N_{S B}} x_{b} \sum_{a=1}^{N_{b}} \mathbf{f}_{a}(\mathbf{r})
$$

\section{Construct Synthetic Basis Function}

The induced current excited from each source configuration constitutes a sampling of the solution space. The orthogonal and complete properties of the auxiliary source are demand for the solution space. A simple way is to consider the configurations of sampling sources obtained from plane wave spectrum surrounding the target object, which is typical of CBFM. In SBFM, the source is defined on the enclosed surface, where the current is discrete in form of RWG functions. Then, the major information on the solution space is extracted with minimum number of degree of freedom(DOF)[3].

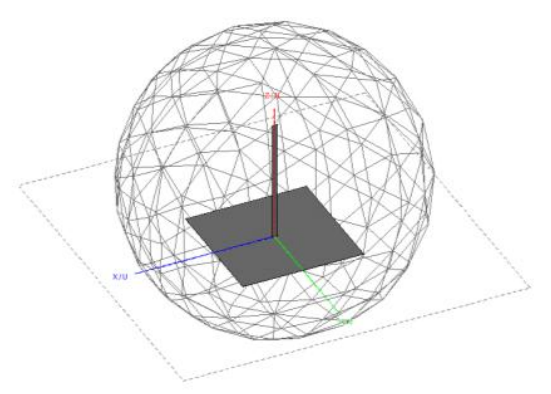

Figure 1. antenna and auxiliary sources model.

For block b, the exciting voltage can be divided into two categories: 1) the incident field or excitation current on each isolating block. 2) the auxiliary sources due to the field radiated by the currents that the external field induces on other blocks. Fig. 1 displays the isolated antenna and a series of auxiliary sources model. Voltage gap source is added between the monopole and plane.

$$
\left\{\begin{array}{l}
{\left[I^{1}\right]_{n_{b} \times 1}=[Z]_{n_{b} \times n_{b}}^{-1}[V]_{n_{b} \times 1}^{1}} \\
{\left[I^{s}\right]_{n_{b} \times n_{s}}=[Z]_{n_{b} \times n_{b}}^{-1}[V]_{n_{b} \times n_{s}}^{s}}
\end{array}\right.
$$

Where the voltage excitation is obtained by

$$
[V]_{n_{s} \times 1}^{1}=\left\langle V \delta(n), \mathbf{f}_{n}\right\rangle= \begin{cases}l_{n} V & m=n \\ 0 & \text { others }\end{cases}
$$

And $n_{b}$ refers to the number of RWG functions defined on block $b$ and $n_{s}$ is the number of RWG functions defined on the auxiliary sources, which represents the impact from the auxiliary source: 


$$
[V]_{n_{b} \times n_{s}}^{s}=\left\langle L\left(\mathbf{f}_{s}(\mathbf{s})\right), \mathbf{f}_{m}(\mathbf{r})\right\rangle
$$

Thus, we obtain the solution space of the current including both the feeding voltages and the coupling fields from the other elements. Then the number of SBFs is determined by applying the Singular Value Decomposition (SVD) algorithm to eliminate any redundancy among the basis functions [4].

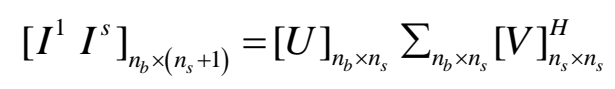

The SBFs are extracted from [U] based on the sequence of $\sum_{n_{b} \times n_{s}}$, and the columns of [U] are the combination of the low order RWG functions. Moreover, if $\rho_{k} / \rho_{1} \leq \rho_{S B F}$, then the expansion coefficients can be written as

$$
[\mathbf{P}]=\left[U_{1}, U_{2}, U_{3}, \ldots, U_{M}\right]
$$

\section{Establish Synthetic Matrix Equation}

The Synthetic Basis Function Method involves the generation of the system matrix, equation solution of which represents the weight coefficients of the SBFs. The matrix equation can be reduced:

$$
\left[Z_{S B F}\right]=[P]^{H}[Z][P]
$$

The excitation vectors are calculated, as follows:

$$
\left[V_{S B F}\right]=[P]^{H}[V]
$$

Consider an antenna array with $\mathrm{M}$ elements. The impedance matrix can be written as:

$$
[Z]_{S B F}=\left(\begin{array}{cccc}
{\left[Z_{S B F}\right]_{1,1}} & {\left[Z_{S B F}\right]_{1,2}} & \ldots & {\left[Z_{S B F}\right]_{1, M}} \\
{\left[Z_{S B F}\right]_{2,1}} & {\left[Z_{S B F}\right]_{2,2}} & \ldots & {\left[Z_{S B F}\right]_{2, M}} \\
\vdots & \ddots & \vdots \\
{\left[Z_{S B F}\right]_{M, 1}} & {\left[Z_{S B F}\right]_{M, 2}} & \ldots & {\left[Z_{S B F}\right]_{M, M}}
\end{array}\right)
$$

The elements of the reduced impedance matrix associated with the SBFM can be generated from the elements of the conventional Method of Moments matrix that has been obtained by using low order basis functions. It should be pointed out that the reduced matrix constructed by SBFM enables us to relax the memory requirements significantly. Compared with the conventional MoM memory cost of $\mathrm{O}\left(M N_{b}^{2}\right)$, the above approach reduce it to $\mathrm{O}\left(M N_{S B F}^{2}\right)$, and $N_{S B F}<<N_{b}$ as mentioned above.

\section{Numerical Results}

To verify the validation of the algorithm, a PEC example composed of 5 sub-monopoles is given here, shown in Fig. 2, was analyzed. The length of the monopole is 0.5 meter, and a PEC plane lies under the bottom. The feeding voltage is imposed at the $\mathrm{T}$ shape junctions. The working frequency is $75 \mathrm{MHz}$. For single block, 370 RWGs are used to mesh the monopole and the plane, added with T junction basis functions. The total number of RWG is 1480 . 


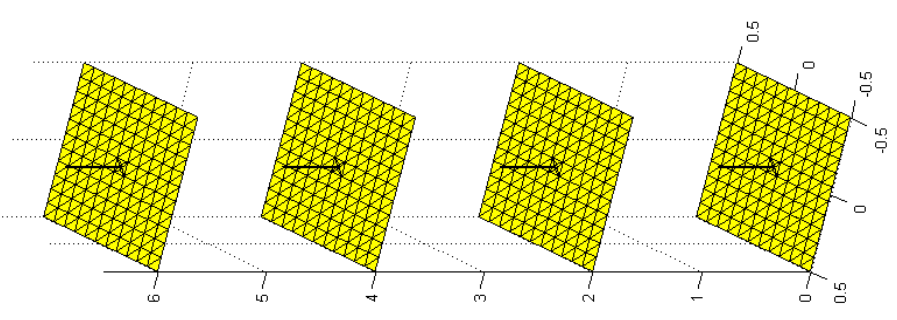

Figure 2. $4 \times 1$ monopole antenna array

The auxiliary source model is a sphere with a radius of $0.8 \mathrm{~m}$. The number of RWG defined on the surface is 123. Set $\rho_{S B F}=0.05$, the total number of SBFs is 230. The results of CST, conventional MoM, and SBFM are given for comparison, as is shown in Fig. 3. The total gain calculated by SBFM is $6.2 \mathrm{~dB}$, slightly different from CST that is $6.4 \mathrm{~dB}$.

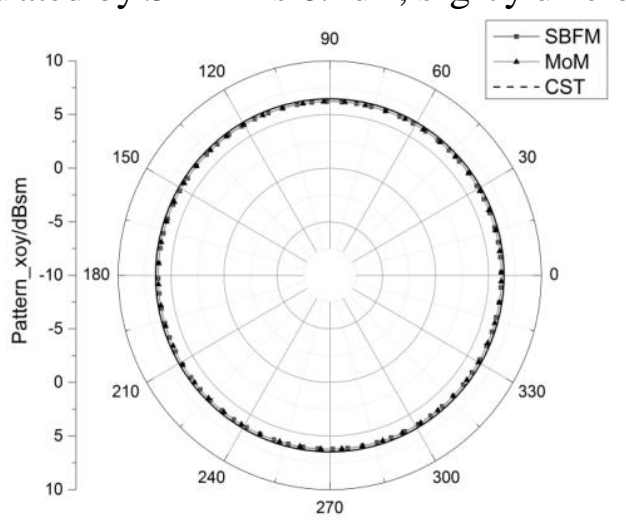

(a)

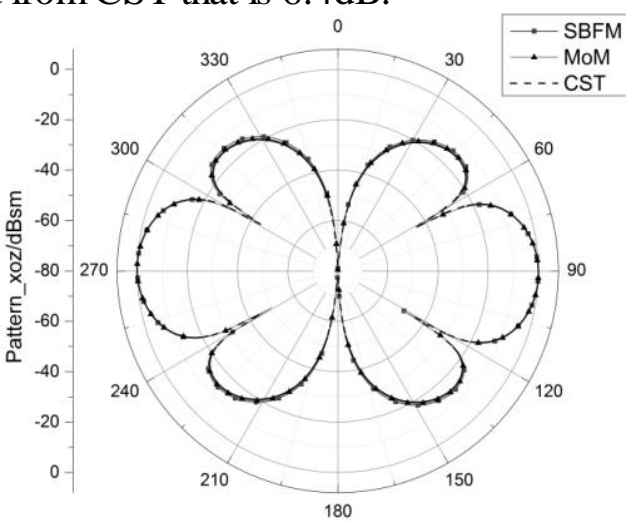

(b)

Figure 3. Properties of MoM, SBFM and CST

The above two results show that SBFM has a good consistency with MoM and CST in solving antenna radiation problem. To evaluate the effects of the error further, we introduce the $\delta$ to express the computational precision.

$$
\delta=\frac{\left\|E_{S B F}-E_{\text {Analysis }}\right\|_{2}}{\left\|E_{\text {Analysis }}\right\|_{2}} \cdot 100 \%
$$

Table 1 gives the computational properties of MoM and SBFM. It is showed that, compared with the MoM, when computational error is $0.683 \mathrm{~dB}$, SBFM reduces the memory cost to $0.13 \%$. Besides, as the scale of the matrix is reduced, efficiency for solving the equation is raised.

Table 1. Properties of MoM and SBFM

\begin{tabular}{ccccc}
\hline & $\begin{array}{c}\text { Number of } \\
\text { unknowns }\end{array}$ & $\begin{array}{c}\text { RAM cost of impedance } \\
\text { matrix[KB] }\end{array}$ & $\begin{array}{c}\text { Total } \\
\text { time[s] }\end{array}$ & $\begin{array}{c}\text { Computational precision } \\
\delta[\mathrm{dB}]\end{array}$ \\
\hline MoM & 1480 & 57979 & 5.819 & 0 \\
\hline SBFM & 230 & 77 & 5.197 & 0.683 \\
\hline
\end{tabular}




\section{Conclusions}

Electromagnetic radiation properties of the antenna array are analysis based on the theory of SBFM and the result is validated with conventional MoM. Therefore, SBFM has significant properties in analyzing large scale antenna array, which traditional MoM hardly deals with because of the immense memory cost. However, SBFM does not accelerate the process of calculating the matrix-vector production, contributing little in efficiency. Further research on combination of fast algorithm is underway.

\section{References}

[1] Harrington R F. The Method of Moments in Electromagnetics[J]. Journal of Electromagnetic Waves \& Applications, 1987, volume 1(3):181-200.

[2] Xu Y, Yang H, Yu W. Scattering Analysis of Periodic Composite Metallic and Dielectric Structures with Synthetic Basis Functions[J]. Applied Computational Electromagnetics Society Journal, 2015, 30(10):1059-1067.

[3] Bucci O M, Franceschetti G. On the degrees of freedom of scattered fields[J]. IEEE Transactions on Antennas \& Propagation, 1989, 37(7):918-926.

[4] Matekovits L, Vecchi G, Dassano G, et al. Synthetic function analysis of large printed structures: the solution space sampling approach[C]. Antennas and Propagation Society International Symposium. 2000:568-571 vol.2. 